\title{
Quantification of chemiluminescent signals using photon-sensitive films or a CCD camera
}

\author{
Claudia Fournier, Stephan Brons, and Gisela Taucher-Scholz \\ Gesellschaft fuer Schwerionenforschung, Damstadt, Germany
}

BioTechniques 35:284-290 (August 2003)

\section{INTRODUCTION}

Changing the abundances of signaling proteins is a common mechanism for the regulation of the cell response to any kind of insult (for review, see References 1 and 2). In addition to posttranslational modifications and altered degradation or stabilization, changes in the synthesis rate of the proteins are often involved in cellular signal transduction. The investigation of signaling pathways requires exact quantification methods, which allows for the determination of the intracellular modulation of proteins in response to genotoxic or cytotoxic stress. The quantitative evaluation of Western blot signals is an experimental procedure commonly employed to assess the relative amounts of proteins (3-5). This approach comprises many steps and is subject to potential fluctuations resulting from nonuniform protein transfer efficiencies from the gel to the membrane and deviations from proportionality in the binding of antibodies. In addition to the optimization of these factors, further quantification requires sensitivity and linearity of the detection system to get reproducible results.

Different detection systems are available for the quantification of chemiluminescent protein signals generated in a Western blotting/immunodetection procedure. Densitometric analysis of signals induced by the emitted photons on films is a commonly used method. Charge-coupled device (CCD) cameras directly integrating the emitted photons are an alternative. They typically show a larger dynamic range and greater linearity compared to film densitometry, but also a lower sensitivity, which requires much longer exposure times. The sensitivity of direct recording systems is generally limited either for geometrical reasons or due to the low excitation probability of a secondary fluorescent photon signal, which is emitted at a significantly reduced rate compared to the photons directly generated in the chemiluminescent reaction. The lower sensitivity is particularly limiting for the detection of proteins expressed at a low level or when signals are faint due to a limited affinity between antibody and antigen. Appropriate CCD systems, however, are very expensive.

When a qualitative detection of the signals is intended, the blackening of light (photon)-sensitive films is very often the method of choice, because films are inexpensive and easy to handle. Their utility for quantitative analysis, however, is limited because of their nonlinear response. Here we propose to complement this method using the calibration of optical films to provide reliable quantitative information. The only special requirements for the correction procedure are a calibration wedge and the appropriate software, which allows for the establishment and application of correction factors. The resulting system is a highly sensitive detector that records protein signals inexpensively within minutes. The results obtained have been cross-checked using the signals from a cooled CCD camera.

\section{MATERIALS AND METHODS}

Various amounts of two proteins, CDKN1A and $\alpha$-tubulin, were quantified in different amounts of cell lysates by immunoblot analysis with signal detection on exposed films to assess the impact of the proposed calibration on the quantification of the recorded signals of these proteins. The parameters of the electrophoretic separation and blotting of the proteins were optimized for CDKN1A $(21 \mathrm{kDa})$, a signaling protein of low molecular weight. The cellular abundance of this signaling protein is low compared to structural proteins, but the amount can change in response to cellular insult $(6,7)$. In contrast, $\alpha$ tubulin $(50 \mathrm{kDa})$ is a structural protein of high abundance and is supposed to be untouched by the signaling pathways in response to cellular damage. In many Western blot experiments $\alpha$-tubulin serves as an internal loading control.

All lysates were prepared from normal human foreskin fibroblasts (AG1522B and AG1522C; Coriell Institute, Camden, NJ, USA). Although purified proteins are generally utilized for standardization, here it was intended to simulate the usual conditions when investigating the abundance of proteins within cells. Cell culturing was carried out as described elsewhere (8). Cell extracts were prepared from confluent cultures. Cells were harvested by trypsinization, centrifuged at $10,000 \times \mathrm{g}$, and pelleted at $4^{\circ} \mathrm{C}$, rinsed in phosphatebuffered saline (PBS), and repelleted. Cells were lysed in chilled radioimmunoprecipitation assay (RIPA) buffer, as described elsewhere (9), and sheared mechanically. The protein content of the supernatant obtained after centrifugation $(10,000 \times g)$ was determined based on the protein assay according to the Bradford method using Roti ${ }^{\circledR}$-Quant, (Carl Roth, Karlsruhe, Germany).

Serial dilutions of the cell extracts with total protein amounts varying between 3 and $100 \mu \mathrm{g}$ were separated in $12 \%$ sodium dodecyl sulfate (SDS)-polyacrylamide gels and then transferred overnight to a polyvinylidene difluoride (PVDF) membrane (Millipore, Bedford, MA, USA) in a wet-transfer system (Bio-Rad, Hercules, CA, USA). The membrane was blocked with $5 \%$ nonfat-dried milk. The membranes were 
probed with monoclonal antibodies in an appropriate dilution directed against specific proteins (anti-CIP1/WAF1; Transduction Laboratories, Lexington, Kentucky, USA; anti- $\alpha$-tubulin clone B-5-1-2; Sigma, St. Louis, MO, USA) followed by a second probing with horseradish peroxidase (HRP)-coupled antibody [sheep anti-mouse immunoglobulin-G (IgG); Amersham Biosciences, Little Chalfont, UK]. HRP is an enzyme catalyzing the chemiluminescent reaction of a substrate (luminol, a cyclic diacylhydrazide), which is commercially available $\left(\mathrm{ECL}^{\circledR}+\right.$ Western blotting detection system; Amersham Biosciences). After addition of the substrate, the emitted photons $(430 \mathrm{~nm})$ were detected quantitatively.

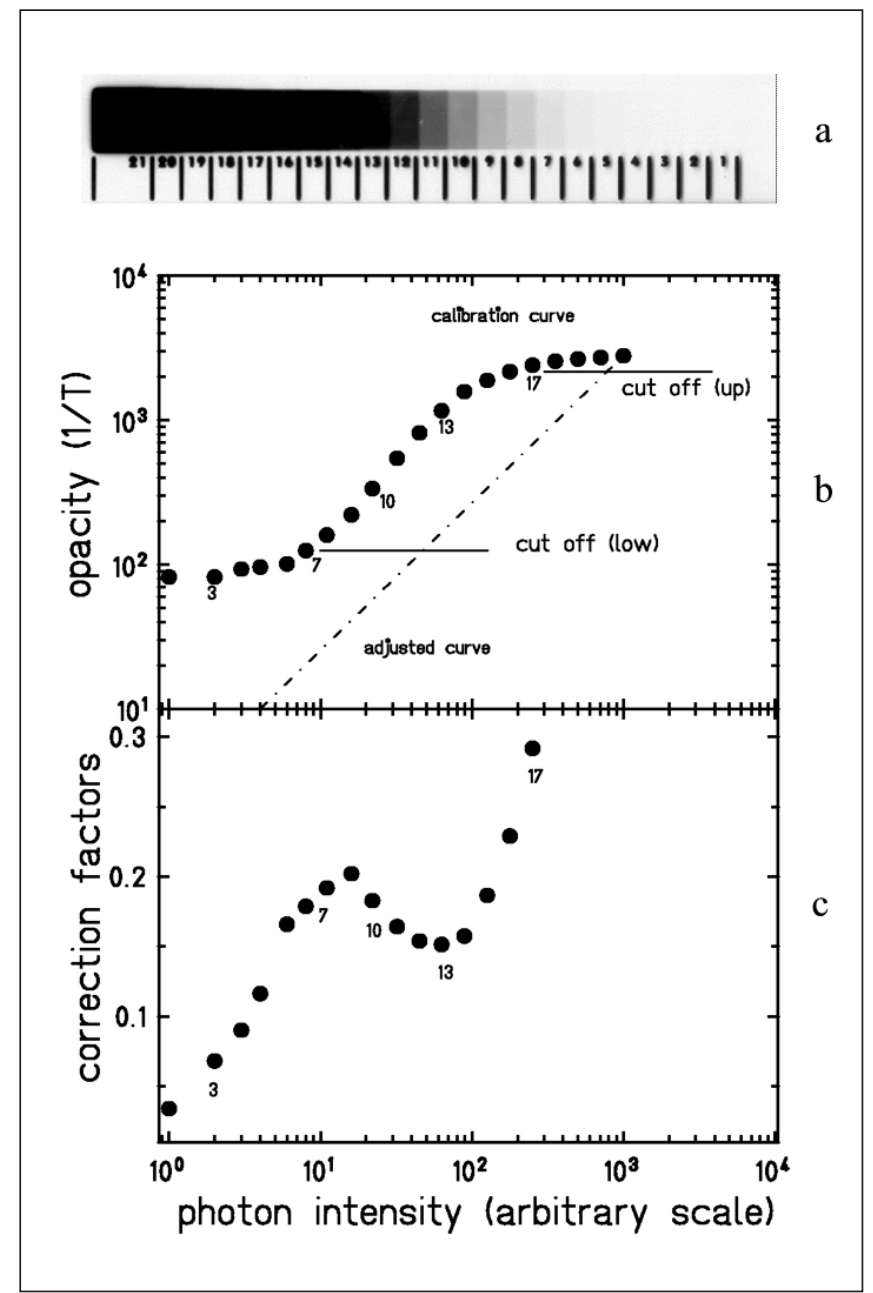

Figure 1. Calibration procedure for densitometric quantification of film signals. (a) Blackening of a film generated with standardized 460-nm light pulses. The steps 1 to 21 correspond to photon intensities of $10^{0}$ to $10^{3}$ on an arbitrary scale, respectively. (b) Calibration curve (black dots) generated by exposing the film to standardized 460-nm light pulses. Signals obtained from light absorption in the induced blackened areas were recorded on a laser scanner and then related to the amount of photons having induced the blackening. The lag phase, saturation level, magnitude, and slope of the calibration curve depend on the sensitivity of the film and the conditions of development. The ideal curve (dashed line) corresponds to a linear relation between the blackening level and photon intensity. A correction function is then generated from the calibration and the ideal curve. We propose the application of a low and a high cut-off level (as indicated), indicating the range of significant measurements. (c) Correction function correcting for the nonlinearity of the film. 


\section{Quantitative Detection of the Chemiluminescent Signals}

Directly after the onset of the chemiluminescent reaction, the membranes were exposed to a photon-sensitive film (Hyperfilm ${ }^{\mathrm{TM}}$ ECL; Amersham BioSciences). The exposure times typically ranged between $30 \mathrm{~s}$ and $5 \mathrm{~min}$. In a second step, each film was calibrated by exposing a border region of the film to standardized blue light pulses of $460 \mathrm{~nm}$ wavelength using a standardized calibration wedge (Densonorm 21, Pehamed; Geräte GmbH, Hattert, Germany) (see Figure 1a). Such a sensitometric calibration is commonly used in radiology to verify the conditions of film development (10). It is carried out within minutes and does not significantly increase the costs of the procedure.

The calibration wedge produces blue light pulses comprising 21 levels of stepwise increasing intensities spanning over 3 orders of magnitude. The lowest photon intensity was arbitrarily set to $10^{0}$. After development of the film, the blackening signals induced by the calibration pulses were determined using the FIPS ${ }^{\circledR}$ Plus laser-densitometer (PTW-Freiburg, Freiburg, Germany). The densitometer recorded the transmission (T) through the blackened areas. A calibration curve was generated by relating the opacity of each calibration signal (opacity $=1 / T$ ) to the level of photons having induced it. Figure $1 \mathrm{~b}$ exemplarily shows such a calibration curve together with a perfectly linear response curve (adjusted here to the value of the maximum photon intensity). The correction for the nonlinearity of the measured values for a given photon intensity was accomplished by folding both curves, and the resulting correction factors were tabulated (see Figure 1c). The film was then scanned a second time, and the correction factors were applied automatically to each pixel of the scanned film image, yielding a corrected image where the blackening of each pixel is now linearly related to the amount of light (photons) having induced it. The corrected images were stored on a disk in a (8bit) tagged image file format (TIFF) file. All these steps were performed automatically using the MEPHYSTO software package (PTW-Freiburg) provided with the laser-densitometer, but any software package that is able to apply a transformation function could be used. In addition, other less expensive densitometers than the laser-densitometer would also be appropriate. It should be noted that the calibration curves were generated for each film separately, thus simultaneously standardizing the conditions of film development.

Finally, the corrected film images were analyzed using the GeneTools software package (Syngene, Cambridge, UK), which integrated the blackening information of each protein band over the complete signal for the protein and subtracted the background by integrating over a corresponding proteinfree area.

In parallel, a cooled CCD camera (Syngene) was used to directly integrate the photons $(430 \mathrm{~nm})$ emitted by the chemiluminescent reaction over a time interval of typically 5-30 min depending on the intensity of the signals. The dynamic range of the camera covers a magnitude of 65,000 (16 bit), and the response of the camera is supposed to be entirely linear. The measurements from the camera and the film were cross-checked and compared to the values expected from the serial dilutions of the cell extract.
The protein amounts, measured from 3 to $100 \mu \mathrm{g}$ total protein loaded, were normalized to the values at $20 \mu \mathrm{g}$ in order to compare between the quantification with the CCD camera and the films. Data were averaged over 2-6 experiments. The errors were calculated as standard errors of the mean (SEM).

\section{RESULTS AND DISCUSSION}

The densitometric quantification of the standardized photon pulses on a chemiluminescent film is shown in Figure 1b (black dots) drawing the opacity (opacity $=1 / \mathrm{T}=\mathrm{I}_{0} / \mathrm{I}$ ) induced by the photon pulses as a function of their intensities. The opacity is related to the optical density by $\mathrm{OD}=\log \mathrm{T}=$ $\log \mathrm{I} / \mathrm{I}_{0}$ according to Lambert-Beer. The measurements show how the dynamic range of the film is limited by the "lag region" and the "saturation region" (observed at very low and very high photon intensities, respectively). In between, the film response is mostly nonlinear, the extension of the apparently linear region being very small. The dashed line represents the ideal curve based on a linear relation between the blackening level (induced opacity) and the photon intensity. Dividing the measured values by the ideal curve results in a function that corrects for the nonlinearity of the film response within the dynamic range (Figure 1c).

The correction for the nonlinearity of the film response becomes important when the signals to be quantitatively compared span over a range with significantly changing correction factors. This is the case when the amounts of protein to be quantified are very different, however the exposure time of the blot membrane on the film also plays a role. Certainly, a correction is only useful within the dynamic range of the film. As a consequence, we suggest defining cut-off levels at photon intensities of $10^{1}$ and $2 \times 10^{2}$ of the noncorrect-

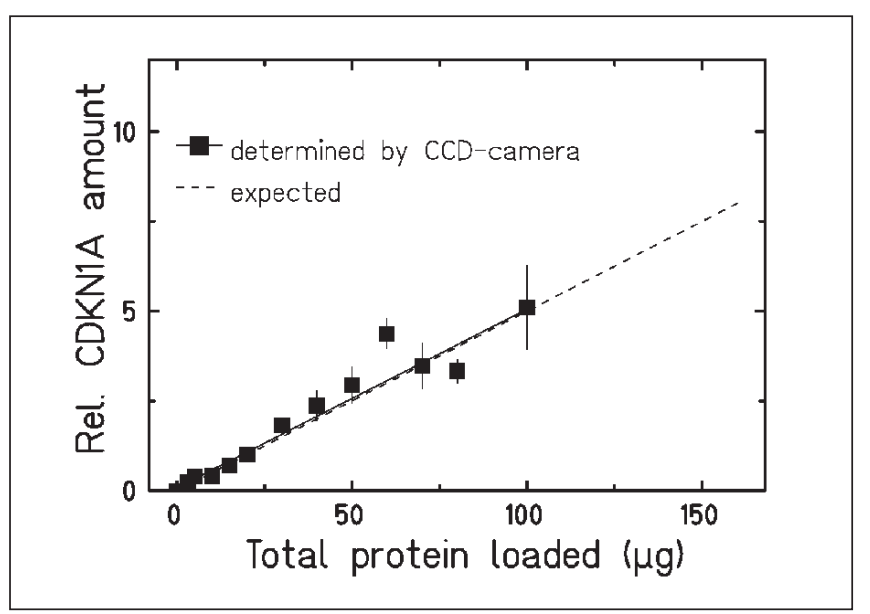

Figure 2. Relative amounts of CDKN1A quantified with a charge-coupled device (CCD) camera and plotted as a function of the amount of total protein loaded on gels. Serial dilutions of cell extracts containing total protein amounts from 3 to $100 \mu \mathrm{g}$ were separated on sodium dodecyl sulfate (SDS)-polyacrylamide gels followed by Western blotting and immunodetection. The results were obtained from six experiments assessing various overlapping ranges of total protein. The linear fit of the data points indicates a good correlation of measured and predicted values: the fitted slope is $1.05 \pm$ $7 \%$ and $\chi^{2} / \mathrm{ndf}$ is 2.2 (ndf $=$ number of degrees of freedom). 


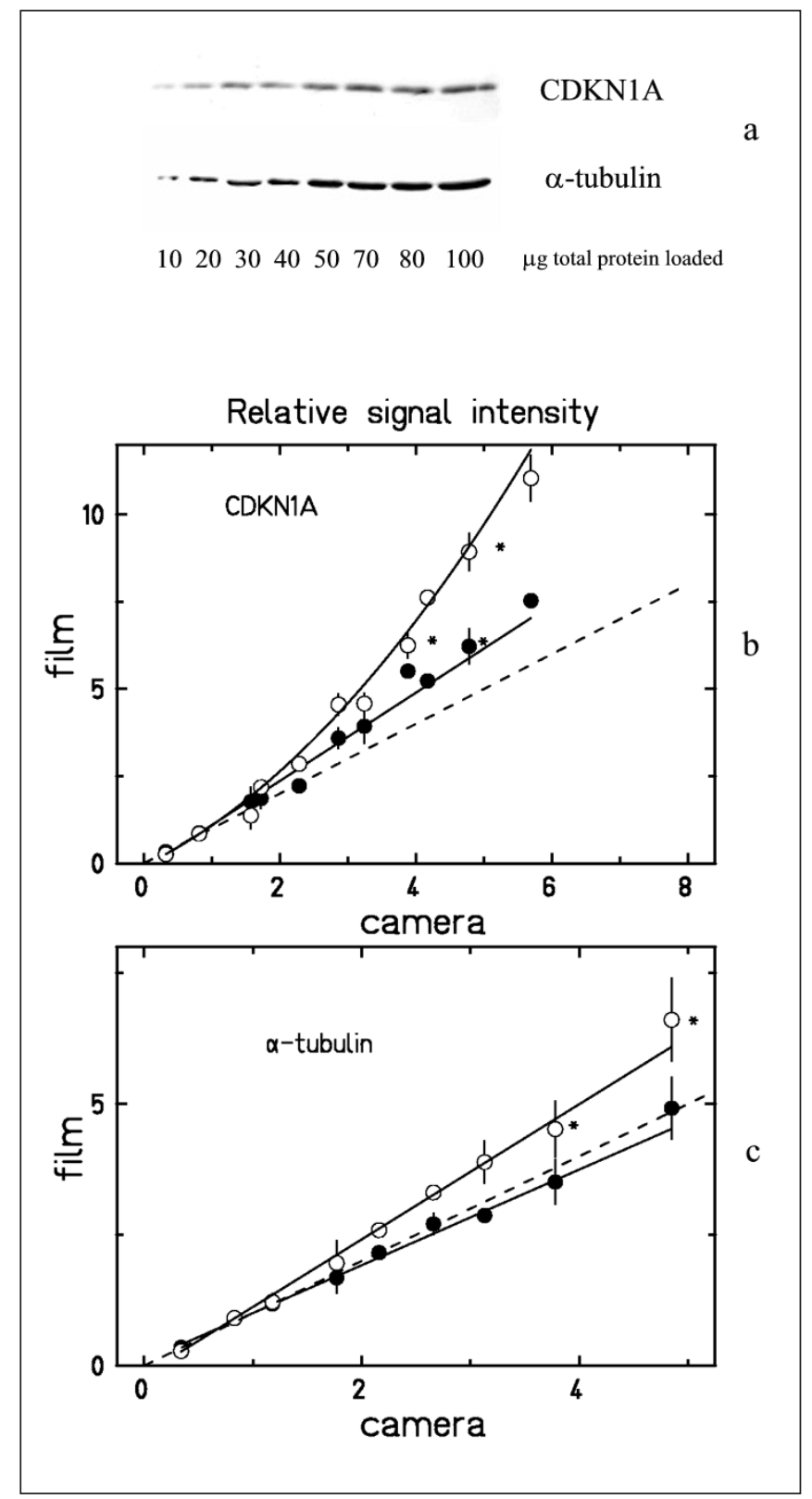

Figure 3. Effect of the correction procedure on protein quantification. (a) Representative Western blot analysis showing corrected signals of protein expression. Aliquots of cell lysates were separated by electrophoresis (10-100 $\mu \mathrm{g}$ total protein/lane) and blotted. Immunodetection was performed for CDKN1A $(21 \mathrm{kDa})$ and $\alpha$-tubulin $(50 \mathrm{kDa})$. (b and c) Cross-check of the densitometric quantification of photon-induced signals on a chemiluminescent film and the direct recording of the chemiluminescence photons with a cooled charge-coupled device (CCD) camera. Increasing amounts of cell lysates were separated by electrophoresis and transferred to a polyvinylidene difluoride (PVDF) membrane. The subsequent immunodetection was carried out for CDKN1A and $\alpha$-tubulin. The results for the two quantification methods were correlated averaging the camera signals within bins of 0.5 width on the arbitrary $\mathrm{x}$-scale. The variation of the points along $\mathrm{x}$-direction corresponds to the chosen bin width. *, indicates single experiments (error bars result from the assorting of the signals); 0 , without correction; $\bullet$, with correction; ---, proportionality $=1$. The parameters of the linear correlation between camera and film quantification for the corrected and noncorrected fit are $1.3 \pm$ $1.3 \%$ and $1.7 \pm 1.0 \%$ for CDKN1A and $0.91 \pm 4 \%$ and $1.3 \pm 3 \%$ for $\alpha$-tubulin. As can be seen from the graph, the noncorrected CDKN1A data are better fitted with a nonlinear function (2nd-order polynomial). 
ed measurements (Figure 1). The indicated cut-off levels have been taken into consideration for the measurements performed in this study.

To obtain appropriate reference data for protein quantification, we used a CCD camera to detect the photon signals obtained from the serial dilutions of the cell extract. As demonstrated in Figure 2, the measured densitometric values for CDKN1A correlate very well with the loaded amounts of total protein. The slope of the linear fit to the data points is 1.05 . Therefore, the values obtained by the quantification of the
CCD camera signals constitute an appropriate reference for the comparison between uncorrected and corrected film results, allowing to discriminate between problems derived from the quantification of the film signals and other experimental errors. For the values measured for CDKN1A with the CCD camera, the average error of each individual measurement is estimated to be approximately $30 \%$. This value was obtained by dividing the mean relative error by the mean number of experiments per data point. Assuming that the quantification step with the CCD camera does not contribute significantly to the experimental error, this estimated error is expected to reflect the uncertainties of the experimental steps preceding the detection.

To address the question to what extent the film results obtained from the densitometric evaluation are comparable to the data shown in Figure 2, the very same membranes were exposed to a chemiluminescent film, and the signals were quantified as described above. Figure 3 shows the comparison between the relative amounts of protein obtained from the $\mathrm{CCD}$ camera and from the quantification of films. In Figure $3 \mathrm{a}$, the corrected film signals of a representative Western blot for the quantification of CDKN1A and $\alpha$-tubulin are shown. Figure $3 b$ depicts the correlation of the relative signal intensities obtained using the films or the camera for the quantification of CDKN1A. A perfect correlation between the values obtained from both detection methods would yield the dotted curve with a proportionality of 1 . However, the noncorrected CDKN1A data detected with the film (open symbols) showed a considerably steeper increase when fitted using a linear correlation (fitted slope parameter $1.7 \pm 1.6 \%$ ) and could in fact be fitted more adequately with a function reflecting a nonlinear film response (2nd polynomal order). This applies to data in a relevant range from 3 to 100 $\mu \mathrm{g}$ total protein loaded on the gel. The nonlinearity of the uncorrected densitometric values becomes especially relevant when greatly differing amounts of protein are detected, as it is the case when large factors of protein accumulation are measured. Applying the correction for the nonlinearity of the film response (closed symbols) clearly results in significant improvement and reduces the deviation between the corresponding film and CCD camera data to remaining 26\% (fitted slope parameter $1.3 \pm 1.3 \%$ ). The parameters of the fit- 
ted slope give a numerical estimate of the improvement achieved by the correction procedure.

For $\alpha$-tubulin, the correlation of the data obtained by the film and the camera is shown in Figure 3c. Also, this correlation was improved by the calibration procedure as shown previously for the CDKN1A data. Accordingly, the deviation from unity of the slope of the linear fit to the $\alpha$-tubulin data was reduced from $30 \%$ for the noncorrected values to less than $9 \%$ for the corrected values.

Besides the good correlation between both detection methods, the analysis of the $\alpha$-tubulin data shows saturation on a level preceding the detection. This becomes apparent above $30-40 \mu \mathrm{g}$ of total protein loaded (in 2 out of 3 experiments) when the measurements of $\alpha$-tubulin with the CCD camera or the film are compared to the amounts of loaded protein (not shown). The saturation affects both detection methods in the same way, resulting in a good agreement for the measured values. Thus, it is not visible in the direct comparison of the data from the CCD camera and the film (Figure 3c). This saturation effect is likely to be a consequence of the separation, transfer, and immunodetection procedures rather than of signal quantification problems. It must be noted that the membranes for $\alpha$-tubulin protein detection are generated in the same experiments as the CDKN1A signals, and the gel running and blotting conditions had been optimized for CDKN1A. Accordingly, the CDKN1A signals did not show such saturation behavior. From these observations, we learn that, depending on the experimental conditions, the strengths of the emitted light signals and the protein amounts cannot always be assumed to correlate linearly. Therefore, it is important to consider sources of errors arising from experimental steps preceding the quantification. Taken together, the correction of the film signals significantly improved the correlation of the protein data obtained by the film and CCD camera quantification.

Other ways of improving the quantification procedure are conceivable. The use of a slower, lower contrast film could lead to an extension of the dynamic range. On the other hand, longer exposure times would become necessary and could enhance any nonlinearity of the film response. Possible factors playing a role are the limited lifetime of the chemiluminescent signals and the limited diffusion velocity of the substrate towards sites of high chemiluminescent activity. Furthermore, the reciprocity failure of the film would be emphasized.

In addition, preflashing, by pre-exposure to a short homogeneous flash of light, is a common approach to hypersensitize the films (11). As a consequence, weak signals will be shifted towards the medium range of the film response curve. Although considered as a useful method to improve the detection capability of films for weak signals, preflashing has the disadvantage of reducing the extent of the dynamic range. Thus, none of these procedures can substitute for a correction of the nonlinear film response.

\section{CONCLUSION}

We have shown that photon-sensitive films can be calibrated and used for the efficient recording and quantification of Western blot signals. They combine the advantages of high sensitivity, low exposure times, and low costs. The dynamic range of the calibrated films is about 20-fold, which is largely sufficient for most cases of cellular protein inductions.

The correction of the film signals results in a linear correlation of the observed film blackening with the CCD camera signals. This is in contrast to the noncorrected film values, which are related nonlinearly to the reference values. Thus, the agreement between film- and camera-generated data is clearly improved when our correction procedure is employed, with a remaining difference smaller than the estimated mean experimental error of $30 \%$. Therefore, the method described can be applied for the accurate quantification of proteins, provided that the experimental steps preceding the detection have been optimized.

\section{ACKNOWLEDGMENTS}

We thank F. Knauf and P. Sander for technical assistance, B. Spielberger and C. Wiese for helpful discussions, and $G$. Kraft for his encouraging support throughout the study.

\section{REFERENCES}

1.Kohn, K.W. 1999. Molecular interaction map of the mammalian cell cycle control and DNA repair system. Mol. Biol. Cell 10:2703-2734

2.Bartek, J. and J. Lukas. 2001. Mammalian G1- and S-phase checkpoints in response to DNA damage. Curr. Opin. Cell Biol. 13:738-747.

3.Towbin, H., T. Staehelin, and J. Gordon. 1979. Electrophoretic transfer of proteins from polyacrylamide gels to nitrocellulose sheets: procedure and some applications. Proc. Natl. Acad. Sci. USA 76:4350-4354.

4.Hershman, K.M., W.W. Fleming, and D.A. Taylor. 1993. A quantitative method for assessing protein abundance using enhanced chemiluminescence. BioTechniques 15:790-794.

5.Fido, R.J., A.S. Tatham, and P.R. Shewry. 1995. Western blotting analysis. Methods Mol. Biol. 49:423-437.

6.El-Deiry, W.S., T. Tokino, V.E. Velculescu, D.B. Levy, R. Parsons, J.M Trent, D. Lin, W.E. Mercer, et al. 1993. WAF1, a potential mediator of p53 tumor progression. Cell 75:817-825.

7.Harper, J.W., G.R. Adami, N. Wei, K. Keyomarsi, and S.J. Elledge. 1993. The p21 Cdk-interacting protein Cip1 is a potent inhibitor of G1 cyclin-dependent kinases. Cell 75:805-816.

8.Fournier, C., M. Scholz, W.K. Weyrather, H.P. Rodemann, and G. Kraft. 2001. Changes of fibrosis-related parameters after high- and lowLET irradiation of fibroblasts. Int. J. Radiat. Biol. 77:713-722.

9.Fournier, C., C. Wiese, and G. Taucher-Scholz. Accumulation of the cell cycle regulators TP53 and CDKN1A (p21) after low- and high-LET irradiation. Radiat. Res. (In press).

10.Normausschuss Radiologie (NAR) im DIN Deutsches Institut fuer Normung e.V. 1991. Deutsche Industrienorm (DIN) 6868 Teil55. Sicherung der Bildqualität in rõntgendiagnostischen Betrieben. Beuth Verlag GmbH, Berlin, Germany.

11.Laskey, R. Amersham Biosciences Website (http://www4 amershambiosciences.com). Efficient detection of biomolecules by autoradiographie, fluorographie or chemiluminescence. Part I, Review 23. Amersham Biosciences, Little Chalfont, UK.

Received 5 March 2003; accepted 6 May 2003.

Address correspondence to:

Claudia Fournier

Department of Biophysics

Gesellschaft fuer Schwerionenforschung

Planckstr. 1

64291 Damstadt, Germany

e-mail:c.fournier@gsi.de 